\title{
Usefulness of LDAEP to Predict Tolerability to SSRIs in Major Depressive Disorder: A Case Report
}

\author{
Young-Min Park ${ }^{\circledR}$, Seung-Hwan Lee ${ }^{1,2}$ and Eun Jin Park ${ }^{1}$ \\ ${ }^{1}$ Department of Neuropsychiatry, Ilsan Paik Hospital, Inje University College of Medicine, Goyang, Korea \\ ${ }^{2}$ Clinical Emotion and Cognition Research Laboratory, Goyang, Korea
}

We report here a patient with major depressive disorder who experienced severe adverse effects after the administration of SSRIs (serotonin selective reuptake inhibitors) without improvement of his depressive symptoms. These adverse effects disappeared and his depressive symptoms improved after discontinuation of the SSRIs and the administration of tianeptine. The patient exhibited a low value for the loudness dependent of auditory evoked potentials (LDAEP) - 0.14 at baseline, which means that his central serotonergic neurotransmission was already highly active. We assumed that it was this high serotonergic activity that rendered him unresponsive to SSRIs, and brought on him the adverse effects, and that the tianeptine was effective due to the lack of serotonin reuptake inhibitory action. Thus, we suggest that LDAEP can be used to predict an individual patient's tolerability and clinical response to SSRIs in major depression.

Psychiatry Investig 2012;9:80-82

Key Words LDAEP, SSRI, Tianeptine, Tolerability, Treatment response.

\section{INTRODUCTION}

The loudness dependence of auditory evoked potentials (LDAEP) is considered a valid indicator of central serotonergic activity in humans. The LDAEP has been identified as being inversely associated with central serotonergic activity, ${ }^{1}$ with a weak LDAEP reflecting high serotonergic neurotransmission. ${ }^{2}$ It was also found that blood serotonin concentration was negatively correlated with corresponding changes of the LDAEP in patients with major depression. ${ }^{3}$ Serotonergic neurotransmission modulates sensory processing in the primary auditory cortex.,4 Therefore the serotonergic system is well suited for a tonic modulation of auditory cortical processing. ${ }^{3}$ The reactivity of the auditory cortex can be assessed using LDAEP.

Based on these findings, there is a significant correlation between a strong LDAEP indicating low serotonergic function and a favorable response to SSRIs in depressed patients. ${ }^{4,5}$ We hy-

Received: August 10, 2011 Revised: September 28, 2011

Accepted: October 5, 2011 Available online: January 17, 2012

$\triangle$ Correspondence: Young-Min Park, MD, PhD

Department of Neuropsychiatry, Ilsanpaik Hospital, Inje University College of Medicine, 2240 Daehwa-dong, Ilsanseo-gu, Goyang 411-706, Korea

Tel: +82-31-910-7260, Fax: +82-31-910-7268,

E-mail: medipark@hanmail.net

(a) This is an Open Access article distributed under the terms of the Creative Commons Attribution Non-Commercial License (http://creativecommons.org/licenses/by$\mathrm{nc} / 3.0$ ) which permits unrestricted non-commercial use, distribution, and reproduction in any medium, provided the original work is properly cited. pothesized that LDAEP can be used to predict the individual patient's tolerability and clinical response to SSRIs in major depression. We report herein a patient with major depressive disorder and a low baseline LDAEP, who showed low dose SSRI-induced adverse effects and that improved after the discontinuation of SSRIs and the administration of tianeptine.

\section{CASE}

A male with a 5-month history of major depressive disorder visited our hospital due to SSRI-induced adverse effects and a depressive episode (depressed mood, low interest, guilt, agitation, and decreased concentration and appetite). He complained that he always experienced gastrointestinal trouble (e.g., nausea and vomiting), generalized weakness, headache, akathisia, and anxiety when taking SSRIs, and they disappeared completely after he discontinued the SSRIs. He had been treated at the psychiatric outpatient clinic of another hospital for 3 weeks with fluoxetine and escitalopram in sequence. At his first visit to our hospital he had not taken his medication for 1 week, he had a Hamilton Rating Scale for Depression (HAMD) score of 21, a Beck Depression Inventory score of 21, and scored negative in the Mood Disorder Questionnaire. Before the antidepressant treatment, Event -related potential (ERP) using auditory stimulation was measured. Auditory stimulation comprised 1,000 
$\mathrm{Hz}$ stimuli with an interstimulus interval that was randomized between 500 and $900 \mathrm{~ms}$. Tones of 1,000 Hz and 80-ms duration (10-ms rise and 10-ms fall) were presented at five intensities $(55,65,75,85$, and $95 \mathrm{~dB}$ SPL) via headphones. EEG data were recorded from 64 scalp sites using silver/silver-chloride electrodes according to the international 10-20 system (impedance $<10 \mathrm{k} \Omega$ ) and using an Auditory Neuroscan SynAmp amplifier (Compumedics USA, El Paso, TX, USA). For each intensity and for the subject, the $\mathrm{N} 1$ peak and $\mathrm{P} 2$ peak were then determined at the $\mathrm{Fz}, \mathrm{Cz}$, and Pz electrodes. The peak-to-peak N1/P2 amplitudes were calculated for the five stimulus intensities, and the LDAEP was calculated as the linear-regression slope.

His LDAEP was found to be low, at -0.14 , which indicates that his central serotonergic neurotransmission was already highly active. We therefore chose to prescribe an antidepressant unrelated to serotonin- reuptake inhibition. He was initially treated with tianeptine $(12.5 \mathrm{mg})$ and clonazepam $(0.5 \mathrm{mg})$ once daily. With no emergence of previously experienced AEs due to SSRIs, his depressive symptoms had improved substantially by day 21, on which his HAMD score had decreased to 11. After 4weeks of this medication, the patient did not complain of any depressive symptoms, and his HAMD score was 6.

\section{DISCUSSION}

We present a case with severe adverse effects after SSRI therapy. His LDAEP value was very low without medication, reflecting high serotonergic functioning at baseline. We assumed that this patient had a low tolerance to SSRIs and did not respond well to them due to an overincrease of serotonergic activity which was already high. This was suggested by adverse effects appearing whenever he took an SSRI. In contrast, no adverse effects occurred when he took the antidepressant tianeptine, which does not cause serotonin-reuptake inhibition.

LDAEP has been proposed as a biological marker of central serotonergic activity in major depression, with relevance to the clinical response to SSRIs. ${ }^{4-7}$ Juckel et al.(2007) reported that responders to the citalopram treatment (serotonergic drug) were characterized by a strong LDAEP at baseline, and responders to reboxetine (nonserotonergic drug) were characterized by a weak LDAEP at baseline. ${ }^{8}$

We have also reported that a strong LDAEP is related to a favorable response to acute SSRI treatment in generalized anxiety disorder $(\mathrm{GAD}){ }^{9}$ GAD patients with a favorable response to escitalopram treatment are characterized by a stronger pretreatment LDAEP. Some investigators have reported a reduced LDAEP after an acute increase in serotonin levels following the administration of citalopram. ${ }^{10}$ Hence, it can be assumed that major depressive disorder patients with a low tolerability will be characterized by a weaker pretreatment LDAEP.
Tianeptine, an "atypical" antidepressant whose pharmacological action differs from that of the "typical" reuptake-blocking agents, prevents stress-induced dendritic atrophy, improves neurogenesis, reduces apoptosis, and normalizes metabolite levels and the hippocampal volume. ${ }^{11}$ In addition, it decreases the activity of serotonin and its levels in the serotonergic synapses of the central nervous system by increasing serotonin reuptake. ${ }^{12}$ Thus, when SSRI causes adverse effects or when there is a low LDAEP value in patient with major depression, tianeptine or some other non-serotonin-related antidepressant should be prescribed.

We described herein a major depressive disorder patient with a favorable response and tolerability to tianeptine, who was found to have a weak pretreatment LDAEP. Measurement of the LDAEP appears to provide useful clinical information for predicting the tolerability and treatment response to SSRIs in patients with major depressive disorder. However, the limitations of this single case report must be acknowledged. Especially, it is possible that the patient was a poor metabolizer to SSRI. Thus, further investigations with larger samples are needed.

\section{Acknowledgments}

This study was supported by a grant from National Research Foundation of Korea (NRF), funded by Ministry of Education and Science Technology (MEST) (2011-0010562).

\section{REFERENCES}

1. Strobel A, Debener S, Schmidt D, Hünnerkopf R, Lesch KP, Brocke B. Allelic variation in serotonin transporter function associated with the intensity dependence of the auditory evoked potential. Am J Med Genet B Neuropsychiatr Genet 2003;118B:41-47.

2. Juckel G, Gallinat J, Riedel M, Sokullu S, Schulz C, Möller HJ, et al. Serotonergic dysfunction in schizophrenia assessed by the loudness dependence measure of primary auditory cortex evoked activity. Schizophr Res 2003;64:115-124.

3. Hegerl U, Gallinat J, Juckel G. Event-related potentials. Do they reflect central serotonergic neurotransmission and do they predict clinical response to serotonin agonists? J Affect Disord 2001;62:93-100.

4. Gallinat J, Bottlender R, Juckel G, Munke-Puchner A, Stotz G, Kuss HJ, et al. The loudness dependency of the auditory evoked N1/P2-component as a predictor of the acute SSRI response in depression. Psychopharmacology (Berl) 2000;148:404-411.

5. Linka T, Sartory G, Bender S, Gastpar M, Müller BW. The intensity dependence of auditory ERP components in unmedicated patients with major depression and healthy controls. An analysis of group differences. J Affect Disord 2007;103:139-145.

6. Hegerl U, Juckel G. Intensity dependence of auditory evoked potentials as an indicator of central serotonergic neurotransmission: a new hypothesis. Biol Psychiatry 1993;33:173-187.

7. Park YM, Lee SH, Kim S, Bae SM. The loudness dependence of the auditory evoked potential (LDAEP) in schizophrenia, bipolar disorder, major depressive disorder, anxiety disorder, and healthy controls. Prog Neuropsychopharmacol Biol Psychiatry 2010;34:313-316.

8. Juckel G, Pogarell O, Augustin H, Mulert C, Muller-Siecheneder F, Frodl $\mathrm{T}$, et al. Differential prediction of first clinical response to serotonergic and noradrenergic antidepressants using the loudness dependence of auditory evoked potentials in patients with major depressive disorder. J Clin Psychiatry 2007;68:1206-1212.

9. Park YM, Kim DW, Kim S, Im CH, Lee SH. The loudness dependence 
of the auditory evoked potential (LDAEP) as a predictor of the response to escitalopram in patients with generalized anxiety disorder. Psychopharmacology (Berl) 2011;213:625-632.

10. Nathan PJ, Segrave R, Phan KL, O'Neill B, Croft RJ. Direct evidence that acutely enhancing serotonin with the selective serotonin reuptake inhibitor citalopram modulates the loudness dependence of the auditory evoked potential (LDAEP) marker of central serotonin function. Hum
Psychopharmacol 2006;21:47-52.

11. Kasper S, McEwen BS. Neurobiological and clinical effects of the antidepressant tianeptine. CNS Drugs 2008;22:15-26.

12. Uzbay TI. Tianeptine: potential influences on neuroplasticity and novel pharmacological effects. Prog Neuropsychopharmacol Biol Psychiatry 2008;32:915-924. 